\title{
Sustained Increase in Intracellular Calcium Promotes Neuronal Survival
}

\author{
Frank Collins,, ${ }^{1}$ Marc F. Schmidt, ${ }^{2}$ Peter B. Guthrie, ${ }^{2}$ and S. B. Kater ${ }^{2}$ \\ 'Synergen, Incorporated, Boulder, Colorado 80301 and ${ }^{2}$ Department of Anatomy and Neurobiology, Colorado State \\ University, Fort Collins, Colorado 80523
}

\begin{abstract}
Ciliary ganglion neurons, half of which normally suffer developmental death in the embryo, will survive in culture in medium supplemented with depolarizing concentrations of potassium. It is not known how elevated potassium acts inside the cell to promote survival. We report here that depolarizing concentrations of extracellular potassium promote neuronal survival by causing a sustained increase in intracellular calcium. Raising extracellular potassium from 5 to $40 \mathrm{~mm}$, an optimal concentration for survival, caused a sustained increase in intracellular calcium from $250 \mathrm{~nm}$ to greater than $600 \mathrm{nM}$. By $26 \mathrm{hr}$, at which time greater than $90 \%$ of neurons in $5 \mathrm{~mm}$ potassium had died, the calcium concentration of neurons in $\mathbf{4 0} \mathrm{mm}$ potassium was still above $400 \mathrm{~nm}$. Reduction of extracellular potassium from $\mathbf{4 0}$ to 5 $\mathrm{mM}$, which prevents the increase in survival, also reduced intracellular calcium back to rest levels. PN200-110, a dihydropyridine calcium channel blocker that inhibits the survival-promoting effect of elevated potassium, also prevented and reversed the potassium-mediated increase in intracellular calcium. In addition, there was a strong, quantitative correlation between the percentage of neuronal survival and the intracellular calcium concentration over a wide range of extracellular potassium concentrations. These results suggest that elevated potassium opens dihydropyridine-sensitive calcium channels, causing a sustained increase in intracellular calcium that quantitatively determines the number of surviving neurons.
\end{abstract}

During embryonic development, many neuronal populations undergo a period of ontogenetic death, during which approximately half of the neurons initially generated die (Purves, 1988). Ontogenetic death is generally thought to occur because of competition among neurons for limited access to target-derived neurotrophic factors essential for survival (Oppenheim, 1989). The possible influence that electrical activity may have in regulating ontogenetic death is less certain. It is of interest that inhibition of afferent electrical input significantly increases the amount of ontogenetic death in ciliary and sympathetic ganglion neurons (Wright, 1981; Maderdrut et al., 1988; Meriney et al., 1987).

\footnotetext{
Received Dec. 11, 1990; revised Mar. 11, 1991; accepted Mar. 21, 1991.

We thank Darin J. Smith for his excellent work in setting up ciliary ganglion neuronal cultures. We also thank Dr. C. E. Eden, Sandoz Pharmaceutical Corporation, for PN200-110 and Dr. A. Scriabine, Miles Institute for Preclinical Pharmacology, for BAYK8644.

Correspondence should be addressed to Dr. Frank Collins, Director of Neuroscience, Synergen, Incorporated, 1885 33rd Street, Boulder, CO 80301.

Copyright (c) 1991 Society for Neuroscience $0270-6474 / 91 / 112582-06 \$ 03.00 / 0$
}

Essentially all of the neurons that would die during the period of ontogenetic death can be kept alive past this period in vitro in medium supplemented with either neurotrophic factors (Berg, 1984) or depolarizing concentrations of potassium (Collins and Lile, 1989). This supports the proposal that neuronal survival in the embryo could be influenced both by access to neurotrophic factors and by electrically mediated depolarization. It is not known how either of these environmental influences acts inside the cell to promote survival.

Ciliary ganglion neurons are a well-characterized experimental system in which to study the intracellular mechanisms regulating neuronal survival. Approximately half of the neurons generated in the chick embryo ciliary ganglion die between days 9 and 14 of embryonic development (Landmesser and Pilar, 1974). When neurons are isolated from ciliary ganglia during the period of cell death in vivo and placed in culture, greater than $90 \%$ of the neurons die within $18 \mathrm{hr}$ (Bennett and White, 1979; Collins and Lile, 1989). A high proportion of such neurons will survive indefinitely in culture if the medium is supplemented either with specific neurotrophic factors (Schubert et al., 1986; Unsicker et al., 1987; Lin et al., 1990) or with elevated concentrations of potassium (Bennett and White, 1979; Collins and Lile, 1989).

Recent pharmacological evidence indicates that depolarizing concentrations of potassium can promote the survival of such neurons by opening dihydropyridine-sensitive voltage-gated calcium channels (Collins and Lile, 1989). The dihydropyridine calcium channel agonist BAYK8644, which allows L-type voltage-gated calcium channels to open at lower levels of depolarization, significantly reduces the concentrations of potassium required for neuronal survival. Similarly, the dihydropyridine L-type voltage-gated calcium channel blockers PN200-110 and nitrendipine completely inhibit neuronal survival in elevated potassium. These results suggested that elevated calcium, by influx through such channels, may be an intracellular mediator of neuronal survival in high potassium.

It was unclear from the pharmacological results mentioned above whether a transient or a sustained elevation of intracellular calcium was necessary for neuronal survival. It is possible that a transient calcium influx would trigger subsequent, possibly calcium-independent, events leading to increased neuronal survival. Alternatively, it may be necessary to maintain calcium at a sustained, elevated level in order to achieve survival. To determine the nature of the calcium involvement in neuronal survival, we have directly measured intracellular calcium levels over a range of elevated extracellular potassium concentrations in the presence and absence of dihydropyridine calcium channel modulators. 


\section{Materials and Methods}

Materials. Fetal calf serum (FCS) was purchased from Hyclone Laboratories (Logan, UT). Culture media and salt solutions were purchased from Irvine Scientific (Santa Ana, CA). Culture dishes were purchased from Costar (Cambridge, MA). Utility-grade pathogen-free fertile White Leghorn chicken eggs were obtained from Spafas (Roanoke, IL). BAYK8644 was the generous gift of Dr. Alexander Scriabine, Miles Institute for Preclinical Pharmacology. PN200-110 was the generous gift of Dr. C. E. Eden, Sandoz Pharmaceutical Corporation. 3-[4,5Dimethylthiazol-2-yl]-2,5-diphenyltetrazolium bromide (MTT) and other chemicals were from Sigma Chemical Co. (St. Louis, MO).

Cell culture. Plastic 35-mm-diameter tissue culture dishes (Falcon) were prepared with a $2.3-\mathrm{cm}$-diameter cutout, to the bottom of which a glass coverslip was cemented. Culture dishes were exposed to a $1-\mathrm{mg} /$ $\mathrm{ml}$ solution of poly-L-ornithine (Sigma, P-3655) in $10 \mathrm{~mm}$ sodium borate (pH 8.4) overnight at $4^{\circ} \mathrm{C}$, washed in distilled water, and air dried. Dishes were exposed for $5 \mathrm{hr}$ at $4^{\circ} \mathrm{C}$ to the conditioned medium from a parietal yolk sac endoderm cell line (Lehman et al., 1974). Wells were washed twice with culture medium immediately before addition of neurons. Treatment with conditioned medium supported neurite growth (Collins, 1984) and aided identification of neurons, but was not essential for survival.

Chick embryo ciliary ganglia were removed from eggs that had been incubated at $38^{\circ} \mathrm{C}$ in a humidified atmosphere for $8-9 \mathrm{~d}$. The ganglia were chemically dissociated first by exposure to solution A (Hanks' balanced salt solution without divalent cations, containing $10 \mathrm{~mm}$ HEPES buffer, $\mathrm{pH} 7.2$ ) for $10 \mathrm{~min}$ at $37^{\circ} \mathrm{C}$, then by exposure to $0.125 \%$ bactotrypsin 1:250 (Difco, Detroit, MI) in solution A for $12 \mathrm{~min}$ at $37^{\circ} \mathrm{C}$. Trypsin was inactivated by addition of FCS to $10 \%$. After this treatment, ganglia were transferred to $1 \mathrm{ml}$ of solution B [high-glucose Dulbecco's Modified Eagle's Medium (DMEM), without bicarbonate, containing $10 \%$ FCS and 10 mM HEPES, pH 7.2] and mechanically dissociated by trituration approximately 10 times through a glass Pasteur pipette whose opening had been fire polished and constricted to a diameter such that it took $2 \mathrm{sec}$ to fill the pipette after maximal depression of the rubber bulb. The dissociated ganglia were then plated in culture medium (DMEM containing $10 \%$ FCS, $4 \mathrm{~mm}$ glutamine, $60 \mathrm{mg} /$ liter penicillin-G, $25 \mathrm{~mm}$ HEPES, $\mathrm{pH} 7.2$ ) in 100 -mm-diameter tissue culture dishes (up to 40 dissociated ganglia per dish) for $3 \mathrm{hr}$. Preplating separated the nonneuronal cells, which adhere to the dish, from the nerve cells, which do not adhere. After $3 \mathrm{hr}$, the nonadherent nerve cells were collected by centrifugation, resuspended in culture medium, and plated on to the glass coverslip in $500 \mu \mathrm{l}$ at 45,000 nerve cells per culture dish. After $30 \mathrm{~min}$, an additional $1.5 \mathrm{ml}$ of culture medium was added. Dishes were incubated at $37^{\circ} \mathrm{C}$ in a humidified atmosphere containing $7.5 \% \mathrm{CO}_{2}$.

MTT assay. Twenty hours after plating, the culture medium in each dish was replaced with $1 \mathrm{ml}$ of culture medium containing any compounds present up to the time of medium change and the tetrazolium dye MTT $(0.3 \mathrm{mg} / \mathrm{ml}$ final concentration), and incubation continued for an additional $4 \mathrm{hr}$. Then, $1 \mathrm{ml}$ of acid alcohol $(6.7 \mathrm{ml}$ of $12 \mathrm{M} \mathrm{HCl}$ per liter of isopropanol) was added, and the contents of each dish were triturated 30 times to solubilize the dye. The optical density at $570 \mathrm{~nm}$ was determined relative to a $690-\mathrm{nm}$ reference for each dish. Direct neuronal counts indicated that maximal survival (100\%) in the MTT assay corresponded to survival of at least $90 \%$ of the neurons initially plated.

Cell counts. Cells were resuspended using $0.06 \%$ bactotrypsin in solution $\mathrm{A}$ at $37^{\circ} \mathrm{C}$ for $15 \mathrm{~min}$, and neurons were counted in a hemocytometer.

Analysis of intracellular calcium levels. Neurons were loaded with fura-2/acetoxymethyl ester (AM), and intracellular calcium levels were measured microscopically in individual neuronal cell bodies, as previously described (Mattson et al., 1989). Briefly, cells were loaded (1$3 \mathrm{hr}$ after plating) with fura-2/AM (Molecular Probes, Eugene, OR; 1 $\mathrm{mM}$ in dimethylsulfoxide diluted to a final incubation concentration of $2 \mu \mathrm{M}$ ) for $40 \mathrm{~min}$, then washed in solution B and incubated for $20 \mathrm{~min}$ to allow hydrolysis of the ester. Cultures were then set on a temperaturecontrolled heated stage and viewed on a Zeiss ICM microscope with an intensified charge coupled device (CCD) camera (Quantex, Santa Clara, CA); the camera output was fed into a QX7-210 image processing system (Quantex, Santa Clara, CA), where it was converted to a $640 \times 480$ digital image (256 gray levels) and averaged for $540 \mathrm{msec}$ ( 16 frames). The excitation wavelength was determined by a computer-controlled filter wheel that alternated between $350 \pm 10-\mathrm{nm}$ and $380 \pm 10-\mathrm{nm}$ interference filters. Neutral density filters $(0.3 \mathrm{ND})$ were inserted in the excitation path to reduce bleaching of the fura- 2 and prevent saturation of the camera by the fluorescent emission. Random fields of neuronal cell bodies (10-30 cells/field) were viewed in individual dishes to determine pretreatment calcium values. Concentrated solutions of potassium and/or drugs were then added, and calcium measurements were made at different time points after addition. For a typical dish, a single time point consisted of calcium values captured from four to six random fields taken over a 1-min period. The fluorescent emission was filtered with a 495-nm long-pass emission filter. Fluorescent images were captured using each excitation filter; the ratio $(R)$ of the fluorescence intensity [(350-nm image $) /(380-\mathrm{nm}$ image $)]$ was converted to calcium concentration using the formula

$$
\mathrm{Ca}=K_{d} \frac{R-R_{\min }}{R_{\max }-R} \times \frac{F_{0}}{F_{s}}
$$

(Grynkiewicz et al., 1985). For our system, $R_{\min }=0.48, R_{\max }=13.25$, $F_{\mathrm{o}} / F_{s}=10$, and $K_{d}=224 \mathrm{nM}$.

\section{Results}

Raising the extracellular potassium concentration to a level sufficient to promote neuronal survival was associated with a rapid and sustained elevation of intracellular calcium. The intracellular calcium concentration in ciliary ganglion neurons in normal $5 \mathrm{~mm}$ potassium was $245 \pm 5 \mathrm{nM}$ (mean $\pm \mathrm{SEM} ; N=454$ cells). When the extracellular potassium concentration was raised to $40 \mathrm{~mm}$, an optimal concentration for survival (Collins and Lile, 1989), the concentration of intracellular calcium increased rapidly within the first minute to at least $1500 \mathrm{nM}$, then decreased within $15 \mathrm{~min}$ to a sustained concentration of $767 \pm$ $25 \mathrm{~nm}(N=111$ cells; Fig. 1$)$. Intracellular calcium remained elevated for at least $26 \mathrm{hr}$ in the continued presence of $40 \mathrm{~mm}$ potassium (Fig. 1, inset). By $26 \mathrm{hr}$, at which time greater than $90 \%$ of neurons in $5 \mathrm{~mm}$ potassium had died, intracellular calcium levels in $40 \mathrm{~mm}$ potassium had decreased $(439 \pm 19 \mathrm{~nm}$; $N=40$ cells) but were still significantly higher than initial levels in $5 \mathrm{~mm}$ potassium ( $p<0.001$, Mann-Whitney $U$ test). The elevation of intracellular calcium observed in $40 \mathrm{~mm}$ potassium was ion specific and not simply due to an increase in the ionic strength, because a $40-\mathrm{mm}$ increase in sodium concentration had no effect on neuronal survival (Collins and Lile, 1989) or on intracellular calcium levels (data not shown).

The dihydropyridinc L-type calcium channel antagonist PN200-110, which at $50 \mathrm{~nm}$ completely blocks neuronal survival in high potassium (Collins and Lile, 1989), also blocked the sustained elevation of intracellular calcium. When the extracellular potassium concentration was raised to $40 \mathrm{~mm}$ in cultures previously exposed to 50 nм PN200-110 for $10 \mathrm{~min}$, the intracellular calcium concentration increased to about 400 IlM within $30 \mathrm{sec}$, but decreased within $10 \mathrm{~min}$ to control concentrations (Fig. 2). These results suggest that neuronal survival in $40 \mathrm{~mm}$ potassium is prevented when the sustained increase in intracellular calcium is blocked. Interestingly, addition of dihydropyridine calcium channel blockers in normal (5 mM) potassium medium caused a significant decrease in baseline calcium levels from $215 \pm 19 \mathrm{nM}$ to $166 \pm 6 \mathrm{nM}(p<0.03$ Mann-Whitney $U$ test), suggesting that calcium influx through L-type channels contributes to basal calcium levels under normal physiological conditions.

Additional evidence demonstrates that depolarization-induced neuronal survival is dependent upon a sustained, rather than a transient, elevation of intracellular calcium. It is known that high potassium must be continuously present to promote 
Figure 1. The effect of elevated extracellular potassium on intracellular calcium. Raising potassium from $5 \mathrm{~mm}$ (normal medium) to $40 \mathrm{~mm}$ by addition of $\mathrm{KCl}$ at 0 min caused an initial peak followed by a sustained increase in intracellular calcium concentration. Over long time periods (inset), calcium levels decreased slightly but remained significantly elevated in the continuous presence of $40 \mathrm{~mm}$ potassium. Time points for the inset are $30 \mathrm{~min}, 4 \mathrm{hr}, 8 \mathrm{hr}$, and $26 \mathrm{hr}$. Intracellular calcium concentrations are plotted as the mean \pm SEM for 50-70 neurons per time point. The broken line represents the average baseline calcium level in normal $5 \mathrm{~mm}$ potassium medium.

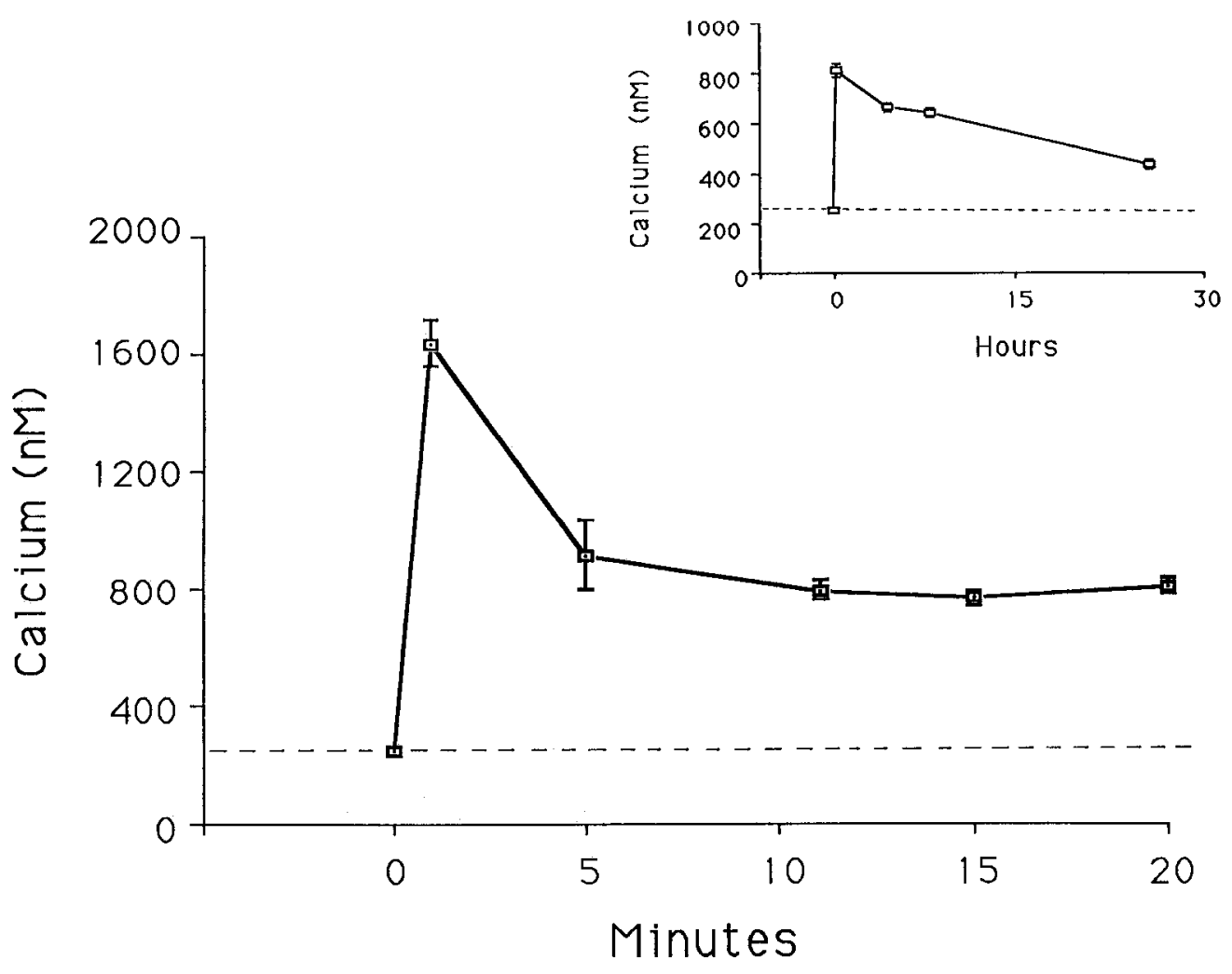

in intracellular calcium, to mean concentrations of $837 \mathrm{~nm}$ and $773 \mathrm{~nm}$, respectively.

\section{Discussion}

Our results demonstrate that elevated extracellular potassium promotes neuronal survival by producing a sustained increase

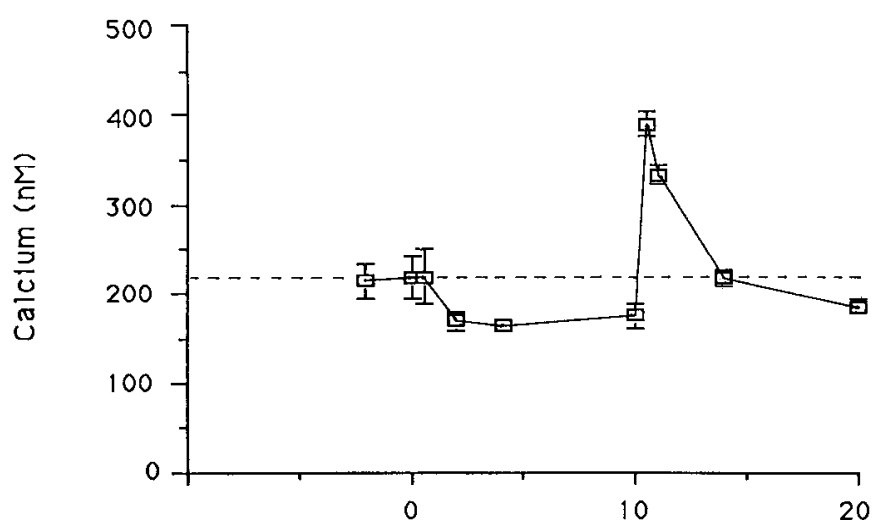

Time $(\mathrm{min})$

Figure 2. Effect of elevated extracellular potassium on intracellular calcium in the presence of the calcium channel blocker PN200-110. Addition of $40 \mathrm{~mm}$ potassium at $10 \mathrm{~min}$ in the presence of $50 \mathrm{~nm}$ PN200110 (added at $0 \mathrm{~min}$ ) prevented the sustained rise in intracellular calcium. Notice that addition of the calcium channel blocker to normal medium ( $5 \mathrm{~mm}$ potassium) in itself produced a small decrease in baseline rest calcium levels (see Results). Intracellular calcium concentrations are plotted as the mean \pm SEM for $50-70$ neurons per time point. The broken line represents the average baseline calcium level in normal 5 mM potassium medium. 
A

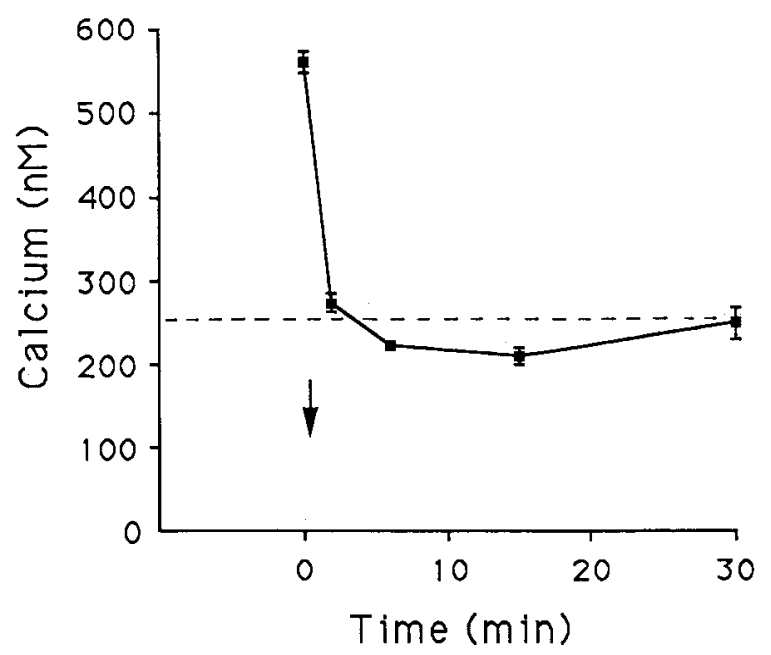

B

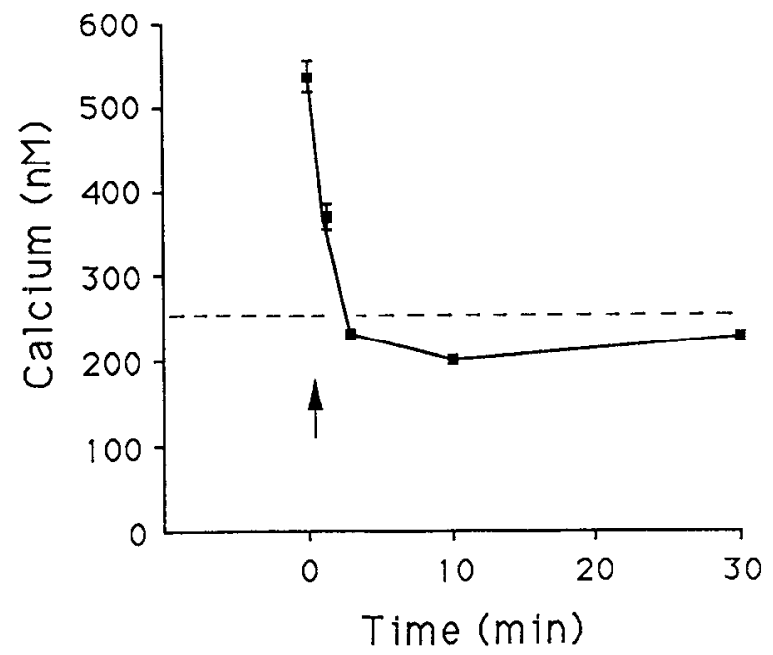

Figure 3. The effect of the withdrawal of high potassium or addition of PN200-1 10 in cultures pretreated with high potassium. Cultures were exposed to $40 \mathrm{~mm}$ potassium for $3 \mathrm{hr}$ before replacement with normal $5 \mathrm{~mm}$ potassium medium (downward arrow in $A$ ), or addition of $50 \mathrm{nM}$ PN200-110 (upward arrow in $B$ ). Intracellular calcium concentrations are plotted as the mean \pm SEM for 35-60 neurons per time point. The broken line represents the average baseline calcium level in normal 5 mM potassium medium.

in the intracellular calcium concentration. Pharmacologic manipulations that prevent or reverse neuronal survival also prevent or reverse the sustained increase in intracellular calcium. In addition, the unanticipated strong quantitative correlation between the percentage of surviving neurons and mean sustained intracellular calcium levels suggests that intracellular calcium levels determine survival.

Our present and previous results (Collins and Lile, 1989) together suggest that increasing extracellular potassium opens dihydropyridine-sensitive L-type voltage-gated calcium channels in chick embryo ciliary ganglion neurons sufficiently to produce a sustained rise in intracellular calcium that is essential for neuronal survival. The promotion of neuronal survival by elevated potassium in chick embryo sympathetic (Collins and Lile, 1989; Koike et al., 1989) and sensory neurons (Collins and

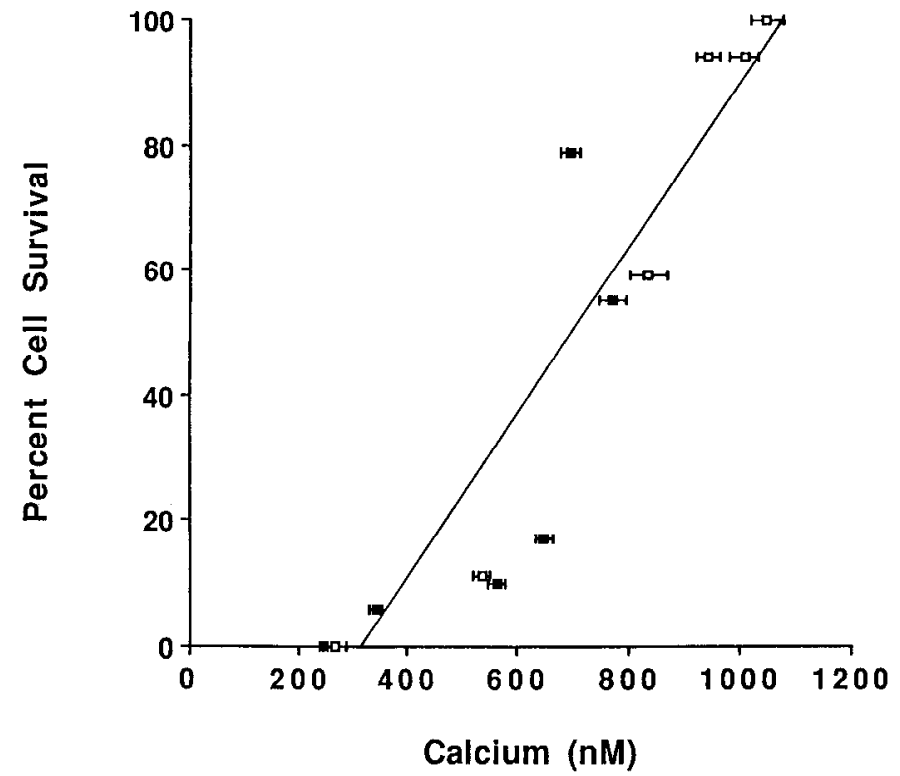

Figure 4. Relationship between the percentage of surviving ciliary ganglion neurons and the intracellular calcium concentration. Intracellular calcium was measured at intervals up to $1 \mathrm{hr}$ after addition of sufficient $\mathrm{KCl}$ to attain final potassium concentrations from 5 to $40 \mathrm{~mm}$ in the presence (open squares) and absence (solid squares) of $50 \mathrm{~nm}$ BAYK 8644 added 20 min before $\mathrm{KCl}$. The stable sustained calcium levels attained by 15 min (see Results) are plotted as the mean \pm SEM for 40-65 cells per observation. The correlation coefficient $(r=0.92)$ was determined for the relation between percent surviving neurons and mean calcium concentration.

Lile, 1989), neonatal rat cerebellar neurons (Gallo et al., 1987), and rat myenteric neurons (Thigpen et al., 1989) has also been demonstrated to depend on the opening of dihydropyridinesensitive voltage-gated calcium channels. Thus, it is likely that elevated intracellular calcium can promote survival in a wide range of different neuronal types. Although it is possible that elevated calcium acts indirectly to promote survival by causing the release of neurotrophic factor(s) from the neurons, the relative insensitivity of potassium-mediated survival to changes in cell density (Collins and Lile, 1989) and the continuous need for potassium and elevated calcium suggest that this explanation is not very likely.

Our results suggest that there is a permissive range of intracellular calcium concentrations compatible with embryonic neuronal survival. This would be in accord with a growing literature implicating calcium as a major developmental regulatory signal. In systems such as the neuronal growth cone, there is a permissive range of intracellular calcium concentrations that is compatible with neurite outgrowth (Kater et al., 1988a). If calcium levels drop too low, neurite elongation ceases. Likewise, in the present study, if calcium levels remain too low, neurons will die. If calcium levels rise too high, neurite elongation also ceases. Likewise, if calcium levels rise too high, for example in response to continuous exposure to the calcium ionophore A23187, even neurons whose survival is promoted by elevated potassium will die (Thigpen et al., 1989).

Although a given range of intracellular calcium concentrations is compatible with survival, the precise range needed for survival may vary between cell types and in a given cell type at different developmental stages. For example, different neurons require different levels of calcium for optimal neurite outgrowth (Kater et al., 1988b; Guthrie et al., 1988), and different cell types 
can respond differently to an equal calcium influx (Mattson et al., 1989b; Mills and Kater, 1990). Variation in the calcium requirements of different neurons may help to explain why some neurons are killed by increased intracellular calcium induced by excitatory amino acids (Kudo and Ogura, 1986; Murphy et al., 1987; Rothman et al., 1987; Kater et al., 1989) or by the HIV viral coat protein gp120 (Dreyer et al., 1990), whereas other neurons, as demonstrated here, are kept alive by a similar increase in intracellular calcium induced by elevated potassium. It may be that embryonic neurons undergoing developmental death in vivo, such as those used in the present study, differ in their calcium requirements for survival from the more mature neurons used to study excitatory amino acid and gp120 neurotoxicity.

The present findings speak to the critical role of intracellular calcium in one of the most fundamental aspects in the establishment and maintenance of neuronal circuits-neuronal survival. To the extent that our results apply to ciliary ganglion neurons in the embryo, they suggest that environmental influences, such as afferent electrical input, that affect intracellular calcium could contribute to the decision of which neurons survive the period of developmental death.

In strong support of this suggestion, it has been demonstrated that blockade of presynaptic electrical input in vivo significantly increases ontogenetic death of nerve cells in the chick embryonic ciliary ganglion (Wright, 1981; Meriney et al., 1987; Maderdrut et al., 1988). Blocking presynaptic electrical input has also been shown to increase neuronal death in other systems, including neurons in the nucleus magnocellularis in young chickens (Born and Rubel, 1988). Thus, presynaptic electrical activity can contribute to neuronal survival. Such activity would transiently depolarize neurons and presumably cause at least a transient elevation in intracellular calcium. Whether this would be sufficient to account for the survival-promoting effects of presynaptic input, as suggested by the results reported here, remains to be determined directly.

It is clear that presynaptic input, even in the ciliary ganglion, is not the only determinant of neuronal survival. It has been demonstrated that neurotrophic factors and postsynaptic target electrical activity both contribute to neuronal survival in the ganglion (Hendry et al., 1988; Meriney et al., 1987). These two factors are probably closely interrelated, because postsynaptic electrical activity has been implicated in regulating neuronal access to target-derived neurotrophic factors at the developing neuromuscular junction (Oppenheim, 1989). With a view to a possible common mechanism of action of pre- and postsynaptic electrical activity on neuronal survival, it would be important to determine whether neurotrophic factors, like neuronal depolarization, promote survival by elevating intracellular calcium or, alternatively, by lowering the range of calcium concentrations required for survival.

\section{References}

Bennett MR, White W (1979) The survival and development of cholinergic neurons in potassium-enriched media. Brain Res 173:549553.

Berg DK (1984) New neuronal growth factors. Annu Rev Neurosci 7: $149-170$.

Born DE, Rubel EW (1988) Afferent influences on brain stem auditory nuclei of the chicken: presynaptic action potentials regulate protein synthesis in nucleus magnocellularis neurons. J Neurosci 8:901-919.

Collins F (1984) An effect of nerve growth factor on the parasympathetic ciliary ganglion. J Neurosci 4:1281-1288.
Collins F, Lile JD (1989) The role of dihydropyridine-sensitive voltage gated calcium channels in potassium mediated neuronal survival Brain Res 502:99-108.

Dreyer EB, Kaiser PK, Offermann JT, Lipton SA (1990) HIV-1 coat protein neurotoxicity prevented by calcium channel antagonists. Science 248:364-367.

Gallo V, Kingsbury A, Balazs R, Jorgensen OS (1987) The role of depolarization in the survival and differentiation of cerebellar granule cells in culture. J Neurosci 7:2203-2213.

Grynkiewicz G, Poenie M, Tsien RY (1985) A new generation of calcium indicators with greatly improved fluorescence properties. J Biol Chem 260:1440-1447.

Guthrie PB, Mattson MP, Mills LR, Kater SB (1988) Calcium homeostasis in molluscan and mammalian neurons: neuron-selective set-point of calcium rest concentrations. Soc Neurosci Abstr 14:582.

Hendry IA, Hill CE, Belford D, Watters DJ (1988) A monoclonal antibody to a parasympathetic neurotrophic factor causes immunoparasympathectomy in mice. Brain Res 475:160-163.

Kater SB, Mattson MP, Cohan C, Connor J (1988a) Calcium regulation of the neuronal growth cone. Trends Neurosci 11:315-321.

Kater SB, Guthrie PB, Mattson MP, Mills LR, Zucker RS (1988b) Calcium homeostasis in molluscan and mammalian neurons: dynamics of calcium regulation. Soc Neurosci Abstr 14:582.

Kater SB, Mattson MP, Guthrie PB (1989) Calcium-induced neuronal degeneration: a normal growth cone regulating signal gone awry? Ann NY Acad Sci 568:252-261.

Koike T, Martin DP, Johnson EM Jr (1989) Role of calcium channels in the ability of membrane depolarization to prevent neuronal death induced by trophic factor deprivation: evidence that levels of internal calcium determine NGF dependence of sympathetic ganglion cells. Proc Natl Acad Sci USA 86:6421-6425.

Kudo Y, Ogura A (1986) Glutamate induced increase in intracellular $\mathrm{Ca}^{2+}$ concentration in isolated hippocampal neurones. Br J Pharmacol 89:191-199.

Landmesser L, Pilar G (1974) Synaptic transmission and cell death during normal ganglionic development. J Physiol (Lond) 241:737749.

Lehman JM, Speers WC, Swartzendruber DE, Pierce GB (1974) Neoplastic differentiation: characteristics of cell lines derived from murine teratocarcinoma. J Cell Physiol 84:13-28.

Lin L-FH, Armes LG, Sommer A, Smith DJ, Collins F (1990) Isolation and characterization of ciliary neurotrophic factor from rabbit sciatic nerves. J Biol Chem 265:8942-8947.

Maderdrut JL, Oppenheim RW, Prevette D (1988) Enhancement of naturally-occurring cell death in the sympathetic and parasympathetic ganglia of the chicken embryo following blockade of ganglionic transmission. Brain Res 444:189-194.

Mattson MP, Guthrie PB, Hayes BC, Kater SB (1989a) Roles for mitotic history in the generation and degeneration of neuroarchitecture. J Neurosci 9:1223-1232.

Mattson MP, Guthrie PB, Kater SB (1989b) A role for Nat dependent calcium extrusion in protection against neuronal excitotoxicity. FASEB J 3:2519-2526.

Meriney SD, Pilar G, Ogawa M, Nunez R (1987) Differential neuronal survival in the avian ciliary ganglion after chronic acetylcholine receptor blockade. J Neurosci 7:3840-3849.

Mills LR, Kater SB (1990) Neuron-specific and state-specific differences in calcium homeostasis regulate the generation and degeneration of neuronal architecture. Neuron 2:149-163.

Murphy SN, Thayer SA, Miller RJ (1987) The effects of excitatory amino acids on intracellular calcium in single mouse striatal neurons in vitro. J Neurosci 7:4145-4158.

Oppenheim RW (1989) The neurotrophic theory and naturally occurring motoneuron death. Trends Neurosci 12:252-255.

Purves D (1988) Body and brain: a trophic theory of neural connections. Cambridge, MA: Harvard UP.

Rothman SM, Thurston JH, Hauhart RE (1987) Delayed neurotoxicity of excitatory amino acids in vitro. Neuroscience 22:471-480.

Schubert D, LaCorbiere M, Esch F (1986) A chick neural retina adhesion and survival molecule is a retinol-binding protein. J Cell Biol 102:2295-2301.

Thigpen JC, Franklin JL, Willard AL (1989) Calcium-dependent effects of acute potassium depolarization on survival of rat myenteric neurons in culture. Soc Neurosci Abstr 15:438.

Unsicker K, Reichert-Preibsch H, Schmidt R, Pettmann B, Labourdette 
G, Sensenbrenner M (1987) Astroglial and fibroblast growth factors have neurotrophic functions for cultured peripheral and central nervous system neurons. Proc Natl Acad Sci USA 84:5459-5463.
Wright L (1981) Cell survival in chick embryo ciliary ganglion is reduced by chronic ganglionic blockade. Dev Brain Res 1:283-286. 